\title{
Increases in alcohol consumption in women and elderly groups: evidence from an epidemiological study
}

Lot M Geels ${ }^{1,3^{*}}$, Jacqueline M Vink ${ }^{1,2}$, Jenny HDA van Beek ${ }^{1,3}$, Meike Bartels ${ }^{1,2,3}$, Gonneke Willemsen ${ }^{1,2,3}$ and Dorret I Boomsma ${ }^{1,2,3}$

\begin{abstract}
Background: In most Western countries, alcohol consumption continues to increase, specifically among women and older adults. Insight into these trends may aid intervention strategies. Here we present data on alcohol consumption by age and sex as well as associations between alcohol use and demographic lifestyle/traits. The data are from a large $(N>16,000)$ population-based Dutch sample, ascertained based on the presence of twins in the family.

Methods: A set of 16 indicators of normative and problematic alcohol use was assessed in participants of the Netherlands Twin Register between 2009-2012 (ages 18-97; 6,052 men; 10,535 women). Alcohol consumption and demographic/lifestyle traits, including educational attainment, work-related/financial stress, urbanization, religiousness, smoking/cannabis initiation, and BMI were described by age and sex. Associations were examined by regressing aspects of alcohol use on age, sex, their interaction, and demographic/lifestyle variables.
\end{abstract}

Results: Age, sex, and initiation of cigarette and cannabis use were the most important predictors of alcohol use. Frequency of alcohol use was lowest between $18-25$ years, with $3.2 \%$ of men and .6\% of women drinking 6-7 times/ week, and highest above age 65 years, with $30.6-32.7 \%$ of men and $20.2-22.0 \%$ of women drinking $6-7$ times/week. Women consumed the lowest quantities of alcohol between 25-45 years, with a 5.7-5.9\% prevalence of excessive drinking (>14 glasses/week), and the largest quantities between 55-65 years (15.5\% excessive drinkers). Age at alcohol initiation, onset of regular drinking, and first alcohol intoxication were lowest between ages 18-25 years and highest above age 65 years. Among older participants, men initiated alcohol use and regular drinking earlier, and had lower age at first intoxication than women, but among young adults, no sex differences were observed.

Conclusions: Alcohol consumption was high in the elderly Dutch population, especially among women. Alcohol initiation, onset of regular drinking, and first alcohol intoxication occur at increasingly younger ages, and the previous gap between men and women in age at alcohol initiation, onset of regular drinking, and first alcohol intoxication has closed almost entirely. Heavy alcohol use was most strongly predicted by older age, sex (male), and initiation of smoking and cannabis use.

Keywords: Alcohol consumption, Young adults, Women, Older adults, Epidemiology, Demographic variables, Lifestyle variables

\footnotetext{
* Correspondence: L.M.Geels@vu.nl

'Department of Biological Psychology, VU University Amsterdam,

Amsterdam, The Netherlands

${ }^{3}$ EMGO+ Institute for Health and Care Research, VU University Medical

Center, Amsterdam, Amsterdam, The Netherlands

Full list of author information is available at the end of the article
} 


\section{Background}

Alcohol use is widespread in the Netherlands, with as much as $88 \%$ of the adult population having consumed alcohol in the past year [1]. The drinking pattern of the Dutch population is characterized by frequent but moderate alcohol consumption [2]. The Dutch drink on average 10 liters of alcohol per person each year, which is slightly higher than the average in the USA ( 9.4 liters) and below that observed across Europe (11 liters) [3,4]. The proportion of recent drinkers (in the last month) is higher in the Netherlands (76\%) than across European countries (67\%) and the USA (52\%) [1,5,6]. Despite the general pattern of moderate drinking, the prevalence of heavy episodic drinking is $8.4 \%$ in Dutch adults $(16 \%$ in men, $5 \%$ in women), which is somewhat higher than in the UK, the USA, and several European countries [4,7]. The proportion of problem drinkers is $9.4 \%$, and the prevalence of alcohol use disorders is $5 \%$ in men and $1 \%$ in women, comparable with other western European countries $[8,9]$. Alcohol use contributes $4.5 \%$ to the total burden of disease in the Netherlands [10], as it increases risk of alcohol abuse and dependency and, when used excessively, contributes to numerous types of disease such as cancer, cardiovascular disease, and liver cirrhosis, as well as to alcohol-related injuries, e.g. traffic accidents [11].

This study presents a detailed assessment of alcohol use in the Dutch adult population, based on data collected between 2009-2011. Trends in alcohol use are examined and risk factors for increased alcohol use are identified based on associations between alcohol use indicators and demographic/lifestyle variables.

Alcohol use typically starts in adolescence, and drinking patterns vary across the lifespan. Before age 16, $72-85 \%$ of Dutch adolescents have initiated alcohol use $[12,13]$. The prevalence of heavy drinking ( $>5$ glasses on at least one occasion weekly) is highest between ages 18-24 [13], and young adults in this age group are at additional risk for alcohol abuse and alcohol-related problems in the college environment [14]. Overall, the prevalence of binge drinking ( $>5$ glasses per occasion) decreases between ages 15 and 64 years [13]. Above age 65, the number of drinkers declines [15]. Across all age groups, the prevalence of alcohol use is higher among men than women, and men have higher incidence of recent drinking and binge drinking [13]. Despite the relatively low number of drinkers above age 65 years, alcohol use disorders in older adults, especially women, seem to be a growing problem in the Netherlands [16]. The gap between men and women is similarly narrowing among young adults; between 2008-2009, the proportion of male heavy drinkers between ages 18-24 decreased substantially (from 37-30\%), while remaining stable among women at $12 \%$ [13].
This age pattern is generally consistent with that observed in several important American studies on the epidemiology of alcohol use. In the USA, the prevalence of alcohol use is highest between age 25-44 and lowest in older age groups $[17,18]$. The college environment similarly puts young adults at additional risk for alcohol abuse and alcohol-related problems [19,20] and among older adults, about 1-3\% are affected by alcohol use disorders [21,22].

As well as age and sex, socioeconomic circumstances and lifestyle factors are related to specific patterns of alcohol use. High educational attainment is associated with higher prevalence of alcohol use, but with lower levels of heavy alcohol use [23]. High levels of workrelated stress have been related to increased quantity of alcohol consumed [24,25]. Financial stress may also be related to alcohol use, as observed in an elderly American sample [26], and in the Netherlands, alcohol dependence is more prevalent among the unemployed and those incapable to work [27]. Alcohol use and binge drinking occur more frequently in rural than in urban areas $[13,28]$, in contrast with the globally observed association of higher alcohol use in urban areas [29]. Religiousness and higher frequency of church attendance are related to lower prevalence of heavy drinking [30-32]. Smoking cigarettes and cannabis use often cooccur with alcohol use $[10,13,33]$. The Netherlands have a unique 'policy of tolerance' towards cannabis, meaning that the substance itself is illegal, but possession of limited amounts of cannabis and selling by licensed establishments is not prosecuted [34].

Physical traits can also predict alcohol use. Body mass index (BMI) may be related to alcohol use through more than one route: in several European countries and the USA, low BMI is related to frequent consumption of small quantities of alcohol and to the preference of wine over beer or strong liquor, whereas high BMI is related to infrequently drinking large quantities of alcohol and preferring strong liquor $[35,36]$.

Detailed insight in the latest trends in alcohol use is valuable to identify groups who are at relatively high risk for alcohol use disorders and to inform intervention strategies for such groups. Specifically, in this study we examine 1) previously observed age and sex effects on alcohol consumption in a large, recently-assessed sample; 2) interaction effects of age and sex on alcohol use; and 3) risk/protective factors for increased alcohol consumption, including demographic/lifestyle traits. These effects are assessed for a large set of normative as well as problematic alcohol variables.

Data were collected between 2009-11 in a large, population-based adult sample of twins (50\%) and nontwins from the Netherlands Twin Register (NTR; $\mathrm{N}=16,587$ ) [37]. Various aspects of alcohol use were 
examined as a function of sex and age-group, namely initiation and frequency of alcohol use, quantity of alcohol consumed, age at initiation and onset of regular drinking, preferred beverage, and situation-specific urges to drink. More severe aspects of alcohol use were also considered, including: number of alcohol intoxications, age at first alcohol intoxication, lifetime alcohol abuse disorder (AAD) symptoms, and hazardous drinking. Associations between alcohol use and age/sex were assessed by regression analysis, with demographic and lifestyle traits: educational attainment, work-related stress, financial stress, degree of urbanization, religiousness, smoking initiation, cannabis initiation, and body mass index (BMI) as covariates.

\section{Methods}

\section{Sample}

Participants were registered with the Netherlands Twin Register (NTR). Adolescent and young adult twins and their parents were initially recruited into the NTR through city councils in the Netherlands [38]. Additional recruitment efforts included the NTR website and yearly newsletter as well as publicity in the media [39]. Longitudinal survey collection in twins and their parents started in 1991. Participants were invited about every 3 years to complete a survey containing questions about health, lifestyle, personality and psychopathology [40]. The present study is based on data from the $8^{\text {th }}$ wave of survey collection that were collected between 2009 and 2011. After obtaining approval from the Medical Ethics Committee of the VU University Medical Center Amsterdam, all NTR participants aged 18 years and older, who were registered at a valid address, were invited to complete the survey $(\mathrm{N}=47,122)$. Participants first received a written invitation including a link to the webpage where they could log on to a web-based version of the survey with a unique, personal login name and password. If subjects did not access the web-based survey in the 6 weeks after the invitation,

\section{Table 1 Age and sex of participants, stratified by family} role

\begin{tabular}{lrrr}
\hline & $\boldsymbol{N}_{\text {ind }}$ & \% women & Mean age (sd) \\
\hline Twins & 8,093 & 68.8 & $33.5(14.5)$ \\
$\begin{array}{l}\text { Higher-order multiples } \\
\text { (triplets/quadruplet) }\end{array}$ & 155 & 70.3 & $25.9(11.9)$ \\
Parents of twins & 5,198 & 59.2 & $55.9(8.1)$ \\
Spouses & 875 & 38.7 & $44.3(11.8)$ \\
Non-twin siblings & 1,898 & 63.4 & $38.3(13.9)$ \\
Other $^{1}$ & 368 & 64.4 & $33.8(10.1)$ \\
Total $^{\text {Tol }}$ & 16,587 & 63.5 & $41.6(16.0)$ \\
\hline
\end{tabular}

${ }^{1}$ includes multiples without information on co-multiples ( $\left.\mathrm{N}=35\right)$, participants registered without any family members $(\mathrm{N}=16)$, spouses of siblings of twins $(\mathrm{N}=28)$, children of twins/siblings $(\mathrm{N}=288)$, and a spouse of a child of a twin $(\mathrm{N}=1)$. they received a paper version of the survey. Between 3-9 months after the paper versions of the survey were sent, subjects who had not responded received a reminder card by post, or a reminder by email (if an email address was available). Several groups of non-responders (e.g. twins from incomplete twin pairs) were reminded in a phone call. The survey was completed by 16,607 individuals (35\% response rate). From these, 20 subjects were removed because they had only completed a small part of the survey. This resulted in a sample of 16,587 subjects from 7,308 families. The average age was $41.6(\mathrm{SD}=16.0$; range $18-97$ ), and $64 \%$ of the participants were women. Participants and their parents were mostly born in the Netherlands (92.7\%). First and second generation immigrants from western countries other than the Netherlands formed $5.6 \%$ of the sample, and $1.7 \%$ were first or second generation immigrants from non-western countries.

Table 1 describes the sample. Twins $(\mathrm{N}=8,093)$, triplets $(\mathrm{N}=154)$, and 1 member of a quadruplet formed the largest group ( $\mathrm{N}=8,248 ; 68.8-70.3 \%$ women), followed by parents $(\mathrm{N}=5,198 ; 59.2 \%$ women), non-twin siblings $(\mathrm{N}=1,898 ; 63.4 \%$ women), and spouses $(\mathrm{N}=875 ; 38.7 \%$ women) of multiples. Twins and higher-order multiples ranged in age between 18-97 years, parents between 30-94 years, non-twin siblings between 18-88 years, and spouses between $25-87$ years.

\section{Measures}

\section{Demographic and lifestyle variables}

Educational attainment was available in 3 categories (primary school/lower vocational schooling, intermediate vocational/upper secondary school', and 'upper vocational/ university'; cf. Statistics Netherlands) [41] for 14,799 subjects. Student status at the time of data collection was based on a single item that asked if subjects were in college/university or high school (0 'no', 1 'yes'). The frequency of work-related stress in the previous year was assessed ('never,' 'occasionally', 'regularly', 'constantly', 'not applicable'), as well as the degree of financial stress ('little/none', 'moderate', 'severe'). Respondents who answered 'not applicable' to the question about work-related stress were included in the category 'never' ( $85 \%$ of these respondents did not have a job).

Degree of urbanization was based on address density in the residential area and measured on a scale of 1 to 5 (very high, high, moderate, low, very low) [42]. Following Statistics Netherlands, degree of urbanization was summarized in two categories (very low - moderate, and heavy - very heavy) [43]. Participants were asked if they were religious, to which they could answer 'no', 'yes, not an active member of church/religious society', or 'yes, active member of church/religious society'.

Subjects were asked if they had ever smoked ('no', 'yes, a few times to try', and 'yes'). The latter two categories were 
collapsed, creating a binary variable that indexed smoking initiation. Participants were also asked if they had ever used cannabis (0 'no', 1 'yes'). BMI was calculated as weight $_{\mathrm{kg}} /$ height $_{\mathrm{m}}^{2}$ and categorized into 'underweight' (BMI <18.5), 'normal weight' (BMI $\geq 18.5 \&<25$ ), and 'overweight' (BMI $\geq 25$ ) [44]. BMI was not computed for women who were pregnant when they completed the survey $(\mathrm{N}=197)$.

\section{Alcohol use}

Participants were asked if they had ever used alcohol ('no', 'a few times to try', and 'yes'). If subjects stated having ever used alcohol, they were asked at what age they had their first drink and at what age they had started drinking regularly (if at all). Subjects were asked how often they had used alcohol in the previous year ('no,' 'monthly or less,' 'two to four times a month,' 'two or three times a week', 'four or five times a week', and 'six times a week or daily'). Quantity of alcohol consumed in the previous year was measured as the number of glasses of beer, wine, and strong liquor, consumed on each day of a normal week. The reported number of glasses was summed into a continuous score for quantity of alcohol consumed per week. This variable was highly positively skewed and therefore categorized into ' 3 glasses or less', '4-7 glasses', '8-14 glasses', '15-21 glasses', and 'more than 21 glasses'. The categories were based on the maximum weekly number of glasses recommended by the Health Council of the Netherlands (7 standard glasses for women, 14 for men), and criteria for excessive alcohol use (over 14 standard glasses per week for women, over 21 for men) $[45,46]$.

Respondents were asked which alcoholic beverage they preferred ('wine,' 'beer,' 'strong liquor,' none'). Urges to drink alcohol in several situations (social situations, during/after dinner, after work, when relaxing, when concentrating, when under stress/pressure) were assessed, based on items about situation-specific urges to smoke [47]. Answer options for each situation were 'not at all,' 'mild', and 'strong'. If participants stated they had ever been intoxicated, they were asked at what age they had been intoxicated for the first time, and the number of times they had been intoxicated. Number of intoxications was severely skewed, and was therefore categorized into 'once or twice, '3-5 times, '6-10 times', '11-25 times', and '>25 times', based on the distribution in the sample. Lifetime prevalence of alcohol abuse and dependence (AAD) symptoms were assessed with the CAGE questionnaire, which consists of 4 items indexing AAD symptoms, that can be answered with 'no,' 'yes, not in the past year' or 'yes, in the past year' [48]. The last two categories were combined, resulting in four binary items for lifetime prevalence of each symptom. Item scores were summed and dichotomized into no (0) versus any (1-4) alcohol abuse and dependence symptoms [49]. Hazardous drinking was indexed by summing the 10 items of the Alcohol Use Disorders Identification Test (AUDIT), and dichotomizing at the cutoff score of 8 , with scores above indicating hazardous drinking [50].

\section{Analyses}

The sample $(\mathrm{N}=16,587)$ was stratified by sex and age (age groups: 18-25, 25-35, 35-45, 45-55, 55-65, $\geq 65$ years). In each sex by age group, the prevalence/ distribution of demographic and lifestyle variables was computed. Subsequently, each case was assigned a proportional weight to correct for the most important deviations from the general population on demographic traits. Alcohol variables were described by sex and age while cases were weighted. For binary/categorical alcohol variables, prevalence/frequency distributions were computed using SPSS 18 [51]. For continuous alcohol variables, mean and standard deviation were computed in Mplus 5 while correcting for familial clustering, since standard deviations are underestimated if subjects are not independent $[52,53]$. Correlations among alcohol use indicators were also corrected for familial clustering in Mplus 5, as were regressions of alcohol use on demographic/lifestyle variables. Data on all predictor variables were available for 12,222 subjects. From this group, $75 \%(\mathrm{~N}=9,103)$ was randomly selected as the main dataset in which the regression analyses were carried out. The remainder of the sample $(\mathrm{N}=3,119)$ was used to cross-validate the results of the regression analyses in. These proportions were chosen to obtain the most reliable estimates of the regression coefficients in the main sample, while retaining a large enough validation sample. Age, sex (0 'male', 1 'female'), an age* sex interaction term, and all other demographic/lifestyle variables were used to predict each aspect of alcohol use. The continuous alcohol measures (age at alcohol initiation, age at onset regular drinking, age at first alcohol intoxication) were predicted with linear regressions, and binary and ordered categorical alcohol measures (frequency of alcohol use, quantity of alcohol consumed, situationspecific urges to drink, number of alcohol intoxications, AAD symptoms, hazardous drinking) were predicted with logistic regressions. Alcohol initiation and preferred beverage were predicted with multinomial regressions, in which the reference categories were 'no' for alcohol initiation and 'no preference' for preferred beverage. The significance level was set at $\alpha=.01$ in the main sample as well as in the validation sample, as a conservative criterion for replication of significant findings. 


\section{Results}

Demographic and lifestyle variables

Table 2 shows prevalence/distribution of demographic and lifestyle factors, stratified by age-group and sex.

The proportion of participants between ages 25-65 with low education was about the same as in the general population ( $23 \%$ versus $27 \%$, respectively), while high education was somewhat more prevalent than in the general population (45\% versus 32\%) [54]. Among participants between $18-25$ years old, the majority (68\%) were students and had not yet completed school or university. This percentage was higher than that observed in the general population (40\%) [55]. Work-related stress (in the previous year) was most prevalent between ages

Table 2 Distribution or prevalence of demographic and lifestyle variables, stratified by age and sex

\begin{tabular}{|c|c|c|c|c|c|c|c|c|c|c|c|c|}
\hline & \multicolumn{2}{|c|}{ Age $18-25$} & \multicolumn{2}{|c|}{ Age 25-35 } & \multicolumn{2}{|c|}{ Age 35-45 } & \multicolumn{2}{|c|}{ Age 45-55 } & \multicolumn{2}{|c|}{ Age 55-65 } & \multicolumn{2}{|c|}{ Age 65 or older } \\
\hline & $\begin{array}{c}\text { Men } \\
N=1,235\end{array}$ & $\begin{array}{c}\text { Women } \\
N=2,499\end{array}$ & $\begin{array}{c}\text { Men } \\
N=839\end{array}$ & $\begin{array}{c}\text { Women } \\
N=1,769\end{array}$ & $\begin{array}{c}\text { Men } \\
N=995\end{array}$ & $\begin{array}{c}\text { Women } \\
N=1,891\end{array}$ & $\begin{array}{c}\text { Men } \\
N=1,192\end{array}$ & $\begin{array}{c}\text { Women } \\
N=2,265\end{array}$ & $\begin{array}{c}\text { Men } \\
N=1,255\end{array}$ & $\begin{array}{c}\text { Women } \\
N=1,507\end{array}$ & $\begin{array}{c}\text { Men } \\
N=536\end{array}$ & $\begin{array}{l}\text { Women } \\
N=604\end{array}$ \\
\hline $\begin{array}{l}\text { Educational } \\
\text { attainment } \\
(N=14,799)\end{array}$ & $\%$ & $\%$ & $\%$ & $\%$ & $\%$ & $\%$ & $\%$ & $\%$ & $\%$ & $\%$ & $\%$ & $\%$ \\
\hline $\begin{array}{l}\text { Primary/lower } \\
\text { vocat. }\end{array}$ & 19.3 & 20.0 & 8.8 & 7.4 & 12.3 & 16.7 & 22.2 & 28.7 & 30.5 & 50.6 & 35.8 & 57.3 \\
\hline $\begin{array}{l}\text { Intermediate } \\
\text { vocat./ upper } \\
\text { sec. }\end{array}$ & 70.2 & 66.5 & 30.8 & 32.2 & 35.5 & 40.4 & 29.5 & 34.4 & 24.8 & 21.5 & 22.5 & 18.6 \\
\hline $\begin{array}{l}\text { Upper vocat./ } \\
\text { university }\end{array}$ & 10.5 & 13.5 & 60.4 & 60.4 & 52.2 & 42.9 & 48.3 & 36.8 & 44.7 & 27.9 & 41.6 & 24.1 \\
\hline \multicolumn{13}{|l|}{$\begin{array}{l}\text { Work-related stress } \\
(N=13,956)\end{array}$} \\
\hline Never & 61.0 & 53.8 & 49.0 & 43.0 & 51.0 & 46.7 & 48.6 & 48.6 & 58.2 & 60.9 & 71.1 & 78.3 \\
\hline Occasionally & 32.7 & 36.8 & 35.6 & 36.8 & 33.1 & 38.0 & 38.8 & 36.1 & 30.9 & 27.7 & 22.1 & 15.8 \\
\hline Regularly & 5.4 & 8.9 & 14.6 & 18.1 & 14.1 & 13.7 & 10.9 & 13.7 & 9.0 & 10.3 & 5.0 & 5.1 \\
\hline Constantly & .9 & .5 & .8 & 2.1 & 1.7 & 1.6 & 1.7 & 1.7 & 1.9 & 1.1 & 1.8 & .8 \\
\hline \multicolumn{13}{|l|}{$\begin{array}{l}\text { Financial stress } \\
(N=14,985)\end{array}$} \\
\hline None/little & 73.8 & 64.6 & 71.7 & 65.5 & 71.7 & 66.8 & 71.2 & 67.2 & 78.5 & 77.4 & 84.6 & 84.4 \\
\hline Moderate & 23.3 & 29.3 & 23.5 & 26.3 & 23.8 & 26.9 & 25.5 & 26.1 & 18.1 & 18.4 & 14.2 & 13.4 \\
\hline Severe & 2.9 & 6.1 & 4.8 & 8.2 & 4.5 & 6.3 & 3.3 & 6.7 & 3.4 & 4.2 & 1.2 & 2.2 \\
\hline \multicolumn{13}{|l|}{$\begin{array}{l}\text { Degree of } \\
\text { urbanization } \\
(N=16,194)\end{array}$} \\
\hline $\begin{array}{l}\text { Urban } \\
\text { residential area }\end{array}$ & 28.7 & 32.4 & 48.4 & 44.1 & 36.1 & 32.0 & 28.3 & 27.6 & 31.9 & 31.6 & 34.2 & 37.3 \\
\hline \multicolumn{13}{|l|}{$\begin{array}{l}\text { Religiousness } \\
(N=16,180)\end{array}$} \\
\hline Not religious & 62.0 & 59.8 & 61.9 & 57.2 & 55.9 & 47.6 & 47.3 & 39.2 & 41.2 & 31.9 & 31.9 & 21.2 \\
\hline $\begin{array}{l}\text { Religious, not } \\
\text { actively }\end{array}$ & 26.7 & 28.9 & 26.2 & 29.1 & 29.6 & 37.4 & 35.1 & 41.7 & 40.1 & 44.9 & 39.1 & 37.8 \\
\hline $\begin{array}{l}\text { Active church } \\
\text { member }\end{array}$ & 11.3 & 11.3 & 12.0 & 13.7 & 14.4 & 15.1 & 17.7 & 19.1 & 18.7 & 23.2 & 29.0 & 40.9 \\
\hline $\begin{array}{l}\text { Smoking initiation } \\
(N=15,527)\end{array}$ & 52.6 & 47.0 & 62.1 & 55.9 & 60.7 & 55.4 & 68.9 & 72.7 & 84.3 & 76.5 & 83.3 & 57.0 \\
\hline $\begin{array}{l}\text { Cannabis initiation } \\
(N=15,122)\end{array}$ & 41.6 & 29.5 & 47.9 & 31.2 & 30.6 & 20.6 & 16.6 & 10.9 & 11.9 & 7.3 & 4.0 & 1.3 \\
\hline \multicolumn{13}{|l|}{$B M I(N=15,889)$} \\
\hline Underweight & 7.7 & 8.8 & 2.2 & 4.6 & .2 & 2.0 & .3 & 1.1 & .2 & 1.3 & .2 & 1.1 \\
\hline $\begin{array}{l}\text { Normal } \\
\text { weight }\end{array}$ & 81.6 & 79.1 & 65.1 & 71.4 & 51.2 & 63.5 & 41.8 & 55.3 & 37.4 & 49.5 & 44.9 & 48.5 \\
\hline Overweight & 10.7 & 12.1 & 32.7 & 24.0 & 48.6 & 34.5 & 57.8 & 43.5 & 62.4 & 49.2 & 54.9 & 50.4 \\
\hline
\end{tabular}


25-35 (51.0\% in men; $57.0 \%$ in women) and least prevalent above age 65 years ( $28.9 \%$ in men, $21.7 \%$ in women). The majority of the participants had experienced little or no financial stress in the previous year (64.6-84.6\%). The proportion of participants who lived in densely populated (urban) areas ranged between $27.6-48.4 \%$ and was somewhat lower than in the general population [43]. Between ages $18-25$, about $40 \%$ of participants was religious (either non-actively; about $28 \%$, or actively; about $11 \%$ ). Religiousness was most prevalent above age 65 years (68.1\% in men, $78.8 \%$ in women). Overall, religiousness was slightly less prevalent than in the general population (about 1-10\% difference) [56].

Between ages 18-25, about half of the participants had initiated smoking cigarettes. Among men, this proportion was highest above age 65 (83.3\%). Among women, the prevalence of smoking initiation was highest between ages 55-65 (76.5\%), but lower above age 65 years $(57.0 \%)$. Overall, the prevalence of smoking initiation was $63.2 \%$, similar to the prevalence in the general population (60.0\%) [57]. Cannabis initiation was most prevalent between ages $25-35$ years $(47.9 \%$ in men, $31.2 \%$ in women), and least prevalent above age 65 years $(4.0 \%$ in men and $1.3 \%$ in women). The prevalence of cannabis initiation in women was highly similar to that in the general population, while in men, it was slightly lower (the largest difference was 9\%) [58]. BMI was higher in the older than in the younger age groups. Above age 45 years, more than half of the men were overweight (54.9-62.4\%). Women were mostly classified in the normal weight range, except above age 55 years, where about $50 \%$ of women were overweight. Overweight was slightly less prevalent than in the general population, mainly in the younger age groups [44].

\section{Alcohol use}

Each case was assigned a proportional weight based on educational attainment, since the sample distribution of this variable deviated most from the distribution in the general population. Table 3 shows prevalence/distribution or mean and standard deviation of alcohol use indicators, stratified by age and sex (see Additional file 1: Table A1 for prevalence/means in the unweighted sample).

Around 94-98\% of the participants had initiated alcohol use, except women aged 65 years or older (85.7\%). Across all age groups, more men had initiated alcohol use than women. Frequency of alcohol use was lowest among young adults (age 18-25 years), with $3.2 \%$ of men and .6\% of women drinking 6-7 times a week. Men above age 65 years drank most frequently (32.7\% drank 6-7 times per week). The proportion of women who drank 6-7 times a week was highest above age 55 years (20.2-22.0\%). Across all age groups, men drank more frequently than women. Quantity of alcohol showed somewhat different age patterns in men and women. In men, drinking more than the recommended 14 glasses per week occurred most often between ages 18-25 years (34.6\%) and nearly as often between ages $55-65$ years (32.4\%). The proportion of men who drank more than 14 glasses a week was smallest between 25-35 years (20.8\%). Excessive drinking (>21 glasses a week) occurred least often in men aged between 35-45 years (9.3\%), and most often between ages 18-25 years (17.2\%). Among women, quantity of alcohol consumed was lowest between ages 25-35 years, with 19.5\% drinking more than the recommended 7 glasses per week, and $5.7 \%$ excessive drinkers ( $>14$ glasses per week). Women drank the largest quantities above age 55 years, with about $40 \%$ drinking more than the recommended 7 glasses per week, and about 15\% excessive drinkers ( $>14$ glasses per week). Men drank larger quantities of alcohol than women across the entire age range.

Wine was more popular above age 65 than in the youngest age group (46.3 versus $2.3 \%$ in men, 85.3 versus $37.3 \%$ in women). Among men, beer was the most popular beverage, while women mostly preferred wine, followed by strong liquor. The urge to drink in social situations was reported most often $(64.1$ - 87.6\%), and the least often reported urge to drink was while concentrating (.9 - 6.5\%). The prevalence of urges to drink in different situations was slightly higher at older ages, especially among women. Generally, men experienced more urges to drink alcohol than women.

The number of alcohol intoxications was highest for individuals between 25-35 years; $26.9 \%$ of men and $8.4 \%$ of women had been intoxicated more than 25 times in this age group, compared to $4.8 \%$ of men and $.8 \%$ of women above age 65 years. Men reported more alcohol intoxications than women. In men, lifetime AAD symptoms were most prevalent between ages 2535 years $(41.4 \%)$ and least prevalent above age 65 years (28.0\%). Among women, lifetime AAD symptoms occurred most frequently between ages 55-65 years (23.3\%), and least frequently above age 65 years (16.2\%). Lifetime prevalence of $\mathrm{AAD}$ symptoms was higher in men than in women. Hazardous drinking occurred most frequently between ages $18-25$ years (29.5\% in men, $13.0 \%$ in women), and least often above age 65 years $(12.7 \%$ in men, $5.5 \%$ in women). Hazardous drinking was more prevalent in men than in women.

Between ages 18-25 years, age at alcohol initiation was lowest, and highly similar for men and women (14.5 in men, 14.6 in women). Among individuals aged 35 years and older, men initiated alcohol use at a younger age than women. Average age at initiation was highest for individuals aged 65 years or older (18.1 years in men and 20.3 in women). Similarly, average age at onset of regular drinking was lowest in the youngest age group (16.7 years in men, 16.8 
Table 3 Prevalence/distribution or mean and standard deviation of alcohol use indicators, by age and sex (subjects weighted for educational attainment)

\begin{tabular}{|c|c|c|c|c|c|c|c|c|c|c|c|c|}
\hline & \multicolumn{2}{|c|}{ Age $18-25$} & \multicolumn{2}{|c|}{ Age 25-35 } & \multicolumn{2}{|c|}{ Age 35-45 } & \multicolumn{2}{|c|}{ Age 45-55 } & \multicolumn{2}{|c|}{ Age 55-65 } & \multicolumn{2}{|c|}{ Age 65 or older } \\
\hline & $\begin{array}{c}\text { Men } \\
N=1,222\end{array}$ & $\begin{array}{c}\text { Women } \\
N=2,493\end{array}$ & $\begin{array}{c}\text { Men } \\
N=829\end{array}$ & $\begin{array}{c}\text { Women } \\
N=1,754\end{array}$ & $\begin{array}{c}\text { Men } \\
N=978\end{array}$ & $\begin{array}{c}\text { Women } \\
N=1,874\end{array}$ & $\begin{array}{c}\text { Men } \\
N=1,201\end{array}$ & $\begin{array}{c}\text { Women } \\
N=2,294\end{array}$ & $\begin{array}{c}\text { Men } N= \\
1,264\end{array}$ & $\begin{array}{c}\text { Women } \\
N=1,523\end{array}$ & $\begin{array}{c}\text { Men } \\
N=543\end{array}$ & $\begin{array}{l}\text { Women } \\
N=612\end{array}$ \\
\hline $\begin{array}{l}\text { Alcohol initiation } \\
(N=16,243)\end{array}$ & $\%$ & $\%$ & $\%$ & $\%$ & $\%$ & $\%$ & $\%$ & $\%$ & $\%$ & $\%$ & $\%$ & $\%$ \\
\hline No & 3.0 & 4.1 & 1.7 & 5.0 & 2.1 & 5.1 & 2.8 & 4.7 & 2.1 & 5.9 & 2.9 & 14.3 \\
\hline A few times to try & 5.4 & 7.7 & 3.2 & 7.2 & 4.9 & 9.2 & 1.8 & 7.1 & 1.7 & 5.8 & 2.5 & 8.7 \\
\hline Yes & 91.6 & 88.2 & 95.1 & 87.7 & 93.0 & 85.7 & 95.4 & 88.2 & 96.1 & 88.4 & 94.7 & 77.0 \\
\hline \multicolumn{13}{|l|}{$\begin{array}{l}\text { Frequency of alcohol } \\
\text { use }(N=15,972)\end{array}$} \\
\hline Never & 7.4 & 10.7 & 6.2 & 18.8 & 7.3 & 16.6 & 6.8 & 13.7 & 5.1 & 14.5 & 9.6 & 23.8 \\
\hline Monthly or less & 13.0 & 28.1 & 14.7 & 33.7 & 16.6 & 28.4 & 9.4 & 19.5 & 9.2 & 16.3 & 9.8 & 15.0 \\
\hline $\begin{array}{l}2-4 \text { times a } \\
\text { month }\end{array}$ & 34.9 & 41.8 & 34.3 & 26.4 & 26.6 & 24.4 & 21.2 & 20.1 & 14.5 & 16.0 & 16.1 & 12.4 \\
\hline 2-3 times a week & 34.1 & 17.1 & 31.9 & 15.4 & 27.7 & 18.0 & 29.3 & 21.6 & 24.3 & 18.7 & 16.3 & 18.0 \\
\hline 4-5 times a week & 7.5 & 1.8 & 8.2 & 3.7 & 10.8 & 6.9 & 14.2 & 10.8 & 16.2 & 12.5 & 15.5 & 10.7 \\
\hline 6-7 times a week & 3.2 & .6 & 4.8 & 2.0 & 11.0 & 5.6 & 19.1 & 14.3 & 30.6 & 22.0 & 32.7 & 20.2 \\
\hline \multicolumn{13}{|l|}{$\begin{array}{l}\text { Weekly alcohol } \\
\text { quantity }(N=12,828)\end{array}$} \\
\hline 3 glasses or less & 22.0 & 39.5 & 23.9 & 52.1 & 24.6 & 46.4 & 20.5 & 35.6 & 14.6 & 29.3 & 17.5 & 30.7 \\
\hline 4-7 glasses & 20.1 & 29.8 & 27.0 & 28.4 & 27.6 & 30.3 & 24.0 & 31.8 & 23.2 & 29.4 & 28.5 & 29.6 \\
\hline 8-14 glasses & 23.4 & 19.1 & 28.2 & 13.8 & 25.7 & 17.4 & 31.5 & 22.3 & 29.9 & 25.8 & 26.4 & 25.4 \\
\hline 15-21 glasses & 17.4 & 6.3 & 10.5 & 3.0 & 12.8 & 3.4 & 13.6 & 7.8 & 18.6 & 10.5 & 15.1 & 11.3 \\
\hline $\begin{array}{l}\text { More than } 21 \\
\text { glasses }\end{array}$ & 17.2 & 5.4 & 10.3 & 2.7 & 9.3 & 2.5 & 10.4 & 2.7 & 13.8 & 5.0 & 12.5 & 3.1 \\
\hline
\end{tabular}

Preferred beverage $(N=14,407)$

Wine

Beer

Strong drinks

No preference

Urges to drink alcohol

Social
situations
$(N=15,052)$

\begin{tabular}{|c|c|c|c|c|c|c|c|c|c|c|c|c|c|}
\hline & Mild & 52.5 & 58.3 & 55.2 & 56.4 & 56.0 & 57.4 & 62.6 & 58.0 & 66.0 & 59.5 & 60.4 & 58.3 \\
\hline & Strong & 27.5 & 18.5 & 32.4 & 20.1 & 25.9 & 15.7 & 18.3 & 11.8 & 12.7 & 8.6 & 7.5 & 58 \\
\hline \multirow{3}{*}{$\begin{array}{l}\text { At dinner } \\
(N=14,968)\end{array}$} & No & 72.8 & 79.6 & 64.0 & 69.8 & 64.7 & 69.0 & 63.6 & 64.7 & 55.7 & 55.5 & 47.7 & \\
\hline & Mild & 26.5 & 19.2 & 33.2 & 26.8 & 31.6 & 27.1 & 32.2 & 31.0 & 39.3 & 40.2 & 46.2 & \\
\hline & Strong & .7 & 1.2 & 2.8 & 3.4 & 3.7 & 3.9 & 4.3 & 4.2 & 5.0 & 4.3 & 6.1 & 3.3 \\
\hline \multirow{3}{*}{$\begin{array}{l}\text { After work } \\
(N=14,828)\end{array}$} & No & 66.1 & 90.5 & 75.4 & 90.5 & 79.7 & 91.2 & 81.3 & 87.7 & 73.9 & 81.3 & 78.0 & \\
\hline & Mild & 26.9 & 8.6 & 21.2 & 7.9 & 17.8 & 7.0 & 15.2 & 9.6 & 22.2 & 15.4 & 18.5 & \\
\hline & Strong & 7.0 & .9 & 3.4 & 1.6 & 2.5 & 1.8 & 3.4 & 2.7 & 3.9 & 3.3 & 3.5 & \\
\hline \multirow{3}{*}{$\begin{array}{l}\text { Relaxing } \\
(N=15,000)\end{array}$} & No & 36.9 & 53.3 & 39.6 & 54.2 & 39.6 & 51.9 & 28.7 & 41.7 & 25.8 & 40.5 & 36.8 & \\
\hline & Mild & 51.5 & 41.7 & 51.7 & 41.8 & 48.8 & 42.6 & 62.1 & 52.5 & 67.3 & 53.9 & 59.5 & \\
\hline & Strong & 11.7 & 5.0 & 8.7 & 4.1 & 11.6 & 5.5 & 9.1 & 5.8 & 6.8 & 5.6 & 3.7 & \\
\hline
\end{tabular}


Table 3 Prevalence/distribution or mean and standard deviation of alcohol use indicators, by age and sex (subjects weighted for educational attainment) (Continued)

\begin{tabular}{|c|c|c|c|c|c|c|c|c|c|c|c|c|c|}
\hline \multirow{3}{*}{$\begin{array}{l}\text { Concentrating } \\
(N=14,667)\end{array}$} & No & 97.3 & 98.3 & 98.0 & 99.0 & 97.4 & 99.1 & 97.6 & 99.0 & 96.3 & 96.5 & 93.5 & 96.3 \\
\hline & Mild & 2.6 & 1.6 & 1.7 & .9 & 2.3 & .8 & 2.3 & 1.0 & 3.4 & 3.3 & 6.3 & 3.7 \\
\hline & Strong & .1 & .1 & .3 & .1 & .3 & .1 & .1 & .1 & .3 & .2 & .2 & 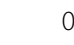 \\
\hline \multirow{3}{*}{$\begin{array}{l}\text { Under stress } \\
(N=14,705)\end{array}$} & No & 79.8 & 84.0 & 80.2 & 84.6 & 82.2 & 82.9 & 78.1 & 78.2 & 74.4 & 72.9 & 75.1 & 73.4 \\
\hline & Mild & 16.4 & 13.0 & 16.2 & 11.9 & 15.5 & 13.9 & 18.0 & 17.6 & 20.6 & 21.7 & 19.9 & 21.2 \\
\hline & Strong & 3.8 & 3.0 & 3.6 & 3.5 & 2.3 & 3.2 & 3.9 & 4.2 & 5.0 & 5.4 & 5.0 & 5.4 \\
\hline \multicolumn{14}{|c|}{$\begin{array}{l}\text { No. of intoxications } \\
(\mathrm{N}=8,885)\end{array}$} \\
\hline \multicolumn{2}{|c|}{ Once or twice } & 29.3 & 39.6 & 14.7 & 32.1 & 18.6 & 37.9 & 22.3 & 44.5 & 25.6 & 50.9 & 36.3 & 55.3 \\
\hline \multicolumn{2}{|l|}{$3-5$ times } & 26.4 & 32.2 & 21.0 & 30.9 & 21.7 & 34.3 & 32.8 & 35.8 & 33.5 & 29.7 & 31.8 & 28.5 \\
\hline \multicolumn{2}{|c|}{$6-10$ times } & 18.3 & 13.7 & 23.2 & 17.1 & 19.9 & 16.2 & 23.9 & 13.1 & 22.0 & 12.1 & 18.0 & 10.6 \\
\hline \multicolumn{2}{|c|}{ 11-25 times } & 13.6 & 7.8 & 14.2 & 11.4 & 16.0 & 7.1 & 10.5 & 3.6 & 10.5 & 4.4 & 9.0 & 4.9 \\
\hline \multicolumn{2}{|c|}{$\begin{array}{l}\text { More than } 25 \\
\text { times }\end{array}$} & 12.5 & 6.7 & 26.9 & 8.4 & 23.8 & 4.5 & 10.5 & 3.0 & 8.4 & 2.9 & 4.8 &. \\
\hline \multicolumn{2}{|c|}{$\begin{array}{l}\text { Alcohol abuse disorder } \\
\text { symptoms }(N=15,227)\end{array}$} & 35.2 & 20.9 & 41.4 & 18.2 & 32.5 & 17.1 & 34.1 & 22.2 & 36.6 & 23.3 & 28.0 & 16.2 \\
\hline \multirow{2}{*}{\multicolumn{2}{|c|}{$\begin{array}{l}\text { Hazardous drinking } \\
(N=15,467)\end{array}$}} & 29.5 & 13.0 & 22.7 & 7.2 & 19.1 & 6.6 & 16.6 & 9.8 & 18.6 & 9.4 & 12.7 & 5.5 \\
\hline & & $\begin{array}{l}\text { mean } \\
(s d)\end{array}$ & $\begin{array}{l}\text { mean } \\
(s d)\end{array}$ & $\begin{array}{l}\text { mean } \\
(s d)\end{array}$ & $\begin{array}{l}\text { mean } \\
(s d)\end{array}$ & $\begin{array}{l}\text { mean } \\
(s d)\end{array}$ & $\begin{array}{l}\text { mean } \\
(s d)\end{array}$ & $\begin{array}{l}\text { mean } \\
(s d)\end{array}$ & $\begin{array}{l}\text { mean } \\
(s d)\end{array}$ & $\begin{array}{l}\text { mean } \\
(s d)\end{array}$ & $\begin{array}{l}\text { mean } \\
(s d)\end{array}$ & $\begin{array}{l}\text { mean } \\
(s d)\end{array}$ & $\begin{array}{l}\text { mean } \\
(s d)\end{array}$ \\
\hline \multirow{2}{*}{\multicolumn{2}{|c|}{$\begin{array}{l}\text { Age at alcohol } \\
\text { initiation }(N=15,155)\end{array}$}} & 14.5 & 14.6 & 15.1 & 15.5 & 15.5 & 16.5 & 15.4 & 16.5 & 16.3 & 17.8 & 18.1 & 20.4 \\
\hline & & (2.0) & (1.7) & (2.4) & $(2.4)$ & (2.5) & (2.9) & $(2.3)$ & (3.0) & (2.8) & $(4.4)$ & $(4.2)$ & (6.8) \\
\hline \multirow{2}{*}{\multicolumn{2}{|c|}{$\begin{array}{l}\text { Age at onset regular } \\
\text { drinking }(N=8,483)\end{array}$}} & 16.7 & 16.9 & 18.2 & 19.6 & 19.1 & 22.6 & 20.4 & 25.5 & 22.6 & 29.6 & 27.8 & 33.3 \\
\hline & & $(1.2)$ & (1.6) & (3.0) & (4.9) & (4.0) & (6.3) & (5.8) & (8.9) & (8.4) & $(11.1)$ & $(12.0)$ & (12.7) \\
\hline \multirow{2}{*}{\multicolumn{2}{|c|}{$\begin{array}{l}\text { Age at first intoxication } \\
(N=10,102)\end{array}$}} & 16.5 & 16.6 & 17.4 & 18.9 & 18.2 & 20.9 & 18.7 & 22.2 & 20.4 & 25.3 & 24.3 & 32.2 \\
\hline & & (1.3) & (1.5) & (2.7) & (3.4) & (3.0) & (5.4) & (3.9) & (7.0) & (5.0) & (8.3) & (8.3) & $(11.0)$ \\
\hline
\end{tabular}

in women) and highest above age 65 years (28.2 years in men, 33.8 in women). Average age at first intoxication showed the same pattern: it was lowest between ages $18-25$ years (16.5 in men, 16.6 in women) and highest above age 65 years (24.2 in men, 31.6 in women). With the exception of the 18-25 age group, men were younger than women at first alcohol intoxication.

Figure 1 shows the correlation coefficients between all indicators of alcohol use, except the nominal alcohol variables (initiation of alcohol use and preferred beverage).

Correlations that were significant at $\alpha=.01$ are shown in black font and non-significant correlations in grey font. Nearly all aspects of alcohol use were significantly interrelated. Age at onset of regular drinking and age at first alcohol intoxication were least strongly associated with other alcohol variables (absolute values of correlations ranged between .00 and .52). Frequency of alcohol use was most strongly related to other alcohol variables (absolute values of correlations ranged between .06 and .70).

\section{Associations of alcohol use with demographic and lifestyle variables}

Associations (standardized regression coefficients; $\beta$ ) between each aspect of alcohol use and demographic and lifestyle variables are shown in Figure 2.

The regression $(\beta)$ coefficients that were significant at $\alpha=.01$ in both the main sample and the validation sample are shown in bold font. The regression coefficients that were significant in the main sample but not in the validation sample are shown in regular font, and the nonsignificant coefficients are shown in grey (see Additional file 2: Table A2 for the cross-validation results). The regression analyses confirmed that age at alcohol initiation, onset of regular drinking, and first alcohol intoxication were lower in younger, than in older participants (betas 


\begin{tabular}{|c|c|c|c|c|c|c|c|c|c|c|c|c|c|}
\hline & 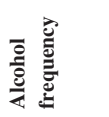 & 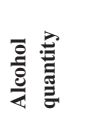 & 氶 & 离 & 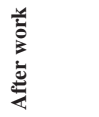 & 先 & 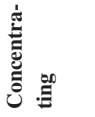 & 总 & 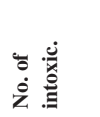 & 悹 & 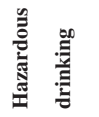 & 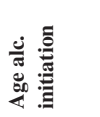 & 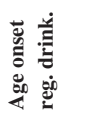 \\
\hline \multicolumn{14}{|l|}{ Alcohol frequency } \\
\hline Alcohol quantity & $.70(.01)$ & & & & & & & & & & & & \\
\hline Social situations & $.40(.01)$ & $.41(.01)$ & & & & & & & & & & & \\
\hline At dinner & $.42(.01)$ & $.29(.01)$ & $.30(.01)$ & & & & & & & & & & \\
\hline After work & $.39(.01)$ & $.39(.01)$ & $.24(.01)$ & $.34(.01)$ & & & & & & & & & \\
\hline When relaxing & $.43(.01)$ & $.40(.01)$ & $.38(.01)$ & $.23(.01)$ & $.27(.01)$ & & & & & & & & \\
\hline Concentrating & $.13(.01)$ & $.15(.01)$ & $.09(.01)$ & $.12(.01)$ & $.20(.02)$ & $.13(.01)$ & & & & & & & \\
\hline Under stress & $.35(.01)$ & $.37(.01)$ & $.27(.01)$ & $.22(.01)$ & $.35(.01)$ & $.31(.01)$ & $.25(.02)$ & & & & & & \\
\hline No. of intoxications & $.19(.01)$ & $.32(.01)$ & $.28(.01)$ & $.11(.01)$ & $.15(.01)$ & $.15(.02)$ & $.04(.01)$ & $.14(.01)$ & & & & & \\
\hline AAD symptoms & $.35(.01)$ & $.42(.01)$ & $.28(.01)$ & $.19(.01)$ & $.26(.01)$ & $.24(.01)$ & $.11(.01)$ & $.33(.01)$ & $.30(.01)$ & & & & \\
\hline Hazard. drinking & $.32(.01)$ & $.46(.01)$ & $.29(.01)$ & $.16(.01)$ & $.30(.01)$ & $.25(.01)$ & $.12(.02)$ & $.33(.01)$ & $.29(.01)$ & $.53(.01)$ & & & \\
\hline Age alc. initiation & $-.06(.01)$ & $-.14(.01)$ & $-.20(.01)$ & $-.04(.01)$ & $-.08(.01)$ & $-.09(.01)$ & $.01(.01)$ & $-.06(.01)$ & $-.25(.02)$ & $-.13(.01)$ & $-.12(.01)$ & & \\
\hline Age onset reg. drink. & $.22(.02)$ & $.03(.01)$ & $-.09(.01)$ & $.11(.01)$ & $.02(.01)$ & $.02(.01)$ & $.03(.01)$ & $.05(.01)$ & $-.21(.01)$ & $.00(.01)$ & $-.03(.01)$ & $.45(.02)$ & \\
\hline Age first intox. & $.13(.01)$ & $-.02(.01)$ & $-.11(.01)$ & $.08(.01)$ & $-.01(.01)$ & $.00(.01)$ & $.02(.01)$ & $.03(.01)$ & $-.31(.01)$ & $-.04(.01)$ & $-.07(.01)$ & $.52(.02)$ & $.52(.02)$ \\
\hline
\end{tabular}

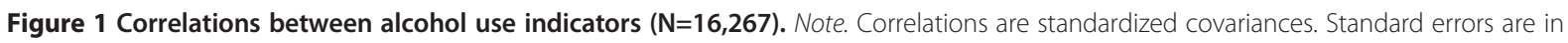
parentheses. Black font: significant at $a=.01$; grey font: non-significant.

between .26 and .36). Older participants drank more frequently and larger quantities of alcohol use, and more often preferred wine (absolute beta values ranged between .11 and .57). Urges to drink alcohol at dinner and under stress were more prevalent among older participants $(\beta=.20$ and $\beta=.12$ respectively). In contrast, the urge to drink in social situations was more prevalent in younger participants $(\beta=-.14)$. Preference for strong liquor decreased over age in women, but this association was not observed in men $(\beta=-.62)$. The urge to drink at dinner was more prevalent in older than in younger women, but in men, no association with age was observed $(\beta=.05)$. Men scored higher than women on nearly all aspects of alcohol use (betas between -.09 and -.24). Men were also

\begin{tabular}{|c|c|c|c|c|c|c|c|c|c|c|c|c|c|}
\hline & age & $\begin{array}{r}\text { sex } \\
(0=\text { male })\end{array}$ & $\operatorname{age} e^{*} \operatorname{sex}$ & $\begin{array}{r}\text { educ. } \\
\text { attainm. }\end{array}$ & student & wrk. stress & fin. stress & urban. & relig & $\begin{array}{l}\text { smoking } \\
\text { initiation }\end{array}$ & $\begin{array}{l}\text { cannabis } \\
\text { initiation }\end{array}$ & bmi & $R^{2}$ \\
\hline \multicolumn{14}{|l|}{ Alcohol inititation $(\mathrm{N}=9,090)$} \\
\hline A few times to try & $-.77(.32)$ & $.08(.17)$ & $.20(.25)$ & $.27(.18)$ & $-.08(.19)$ & $-.49(.19)$ & $.35(.17)$ & $-.06(.16)$ & $.05(.16)$ & $.58(.17)$ & $.20(.32)$ & $-.11(.17)$ & \\
\hline Yes & $.08(.11)$ & $-.21(.05)$ & $-.13(.08)$ & $.35(.06)$ & $.08(.06)$ & $-.09(.06)$ & $.06(.06)$ & $-.01(.05)$ & $-.13(.05)$ & $.62(.06)$ & $.44(.09)$ & $-.10(.06)$ & \\
\hline Alcohol frequency $(\mathrm{N}=9,003)$ & $.29(.02)$ & $-.17(.01)$ & $.02(.02)$ & $.14(.01)$ & $.10(.01)$ & $-.01(.01)$ & $.00(.01)$ & $.00(.01)$ & $-.03(.01)$ & $.18(.01)$ & $.07(.01)$ & $-.09(.01)$ & .17 \\
\hline Alcohol quantity $(\mathrm{N}=7,521)$ & $.11(.02)$ & $-.24(.01)$ & $.05(.02)$ & $.00(.01)$ & $.10(.01)$ & $-.01(.01)$ & $.04(.01)$ & $-.03(.01)$ & $-.07(.01)$ & $.18(.01)$ & $.12(.01)$ & $-.03(.01)$ & .15 \\
\hline \multicolumn{14}{|l|}{ Preferred beverage $(\mathrm{N}=8,301)$} \\
\hline Wine & $.57(.07)$ & $.76(.03)$ & $.01(.06)$ & $.36(.04)$ & $-.04(.05)$ & $.09(.05)$ & $-.08(.05)$ & $-.05(.04)$ & $.04(.04)$ & $.19(.04)$ & $-.03(.05)$ & $-.07(.04)$ & \\
\hline Beer & $-.31(.07)$ & $-.90(.02)$ & $.03(.07)$ & $-.07(.05)$ & $-.06(.05)$ & $.04(.05)$ & $-.03(.06)$ & $-.13(.04)$ & $-.07(.05)$ & $.31(.04)$ & $.09(.05)$ & $-.08(.05)$ & \\
\hline Strong liquor & $-.33(.19)$ & $.33(.11)$ & $-.62(.16)$ & $-.38(.09)$ & $-.19(.11)$ & $.12(.12)$ & $.03(.11)$ & $-.08(.10)$ & $-.09(.11)$ & $.26(.10)$ & $.01(.11)$ & $.34(.10)$ & \\
\hline \multicolumn{14}{|l|}{ Urge to drink alcohol } \\
\hline Sœial situations $(\mathrm{N}=8,625)$ & $-.14(.02)$ & $-.09(.01)$ & $.02(.02)$ & $.14(.01)$ & $.06(.01)$ & $.00(.01)$ & $.02(.01)$ & $.04(.01)$ & $-.02(.01)$ & $.16(.01)$ & $.11(.01)$ & $-.03(.01)$ & .11 \\
\hline At dinner $(\mathrm{N}=8,583)$ & $.20(.02)$ & $-.01(.01)$ & $.05(.02)$ & $.19(.01)$ & $.04(.02)$ & $-.01(.01)$ & $.02(.02)$ & $.10(.01)$ & $.00(.01)$ & $.06(.01)$ & $.07(.01)$ & $-.08(.01)$ & .10 \\
\hline After work $(\mathrm{N}=8,513)$ & $.03(.03)$ & $-.17(.02)$ & $.16(.02)$ & $.01(.02)$ & $.06(.02)$ & $.03(.02)$ & $.03(.02)$ & $.05(.02)$ & $-.07(.02)$ & $.16(.02)$ & $.12(.02)$ & $-.08(.02)$ & .13 \\
\hline When relaxing $(\mathrm{N}=8,599)$ & $.04(.02)$ & $-.13(.01)$ & $.04(.02)$ & $.00(.01)$ & $.02(.02)$ & $.01(.01)$ & $.03(.01)$ & $.00(.01)$ & $.04(.01)$ & $.11(.01)$ & $.03(.01)$ & $-.01(.01)$ & .05 \\
\hline Concentrating $(\mathrm{N}=8,445)$ & $.17(.07)$ & $-.17(.04)$ & $-.01(.07)$ & $-.10(.04)$ & $.03(.06)$ & $-.04(.05)$ & $.15(.04)$ & $.10(.04)$ & $.00(.05)$ & $.27(.06)$ & $.00(.05)$ & $-.12(.04)$ & .18 \\
\hline Understress $(\mathrm{N}=8,461)$ & $.12(.03)$ & $.00(.02)$ & $.08(.02)$ & $.05(.02)$ & $.06(.02)$ & $.03(.02)$ & $.08(.02)$ & $.04(.02)$ & $-.02(.02)$ & $.21(.02)$ & $.11(.02)$ & $-.04(.02)$ & .11 \\
\hline No. of intoxications $(\mathrm{N}=5,216)$ & $-.11(.02)$ & $-.24(.01)$ & $.00(.02)$ & $.09(.01)$ & $-.03(.02)$ & $-.05(.02)$ & $.06(.02)$ & $.04(.01)$ & $-.10(.01)$ & $.09(.01)$ & $.20(.02)$ & $.04(.01)$ & .17 \\
\hline AAD symptoms $(\mathrm{N}=8,745)$ & $.02(.02)$ & $-.15(.01)$ & $.06(.02)$ & $.09(.01)$ & $.06(.02)$ & $.03(.02)$ & $.04(.02)$ & $.02(.01)$ & $-.06(.01)$ & $.18(.02)$ & $.15(.01)$ & $-.03(.01)$ & .13 \\
\hline Hazard. drinking $(\mathrm{N}=8,798)$ & $-.06(.03)$ & $-.19(.02)$ & .07 (.CB) & $.05(.02)$ & $.08(.02)$ & $.04(.02)$ & $.04(.02)$ & $.04(.02)$ & $-.03(.02)$ & $.22(.02)$ & $.14(.02)$ & $-.01(.02)$ & .15 \\
\hline Age alc. initiation $(\mathrm{N}=8,632)$ & $.26(.02)$ & $.12(.01)$ & $.12(.02)$ & $-.05(.01)$ & $-.02(.01)$ & $-.03(.01)$ & $.00(.01)$ & $.03(.01)$ & $.02(.01)$ & $-.17(.01)$ & $-.08(.01)$ & $.00(.01)$ & .18 \\
\hline Age onset reg. drink. $(\mathrm{N}=4,845)$ & $.36(.02)$ & $.19(.01)$ & $.17(.02)$ & $.04(.02)$ & $.02(.01)$ & $-.09(.01)$ & $.03(.01)$ & $.01(.01)$ & $.05(.01)$ & $-.03(.01)$ & $-.03(.01)$ & $-.05(.01)$ & .27 \\
\hline Age first intox. $(\mathrm{N}=5,898)$ & $.32(.02)$ & $.22(.01)$ & $.20(.02)$ & $-.01(.01)$ & $.03(.01)$ & $.01(.01)$ & $-.02(.01)$ & $.05(.01)$ & $.06(.01)$ & $-.09(.01)$ & $-.08(.01)$ & $-.01(.01)$ & .25 \\
\hline
\end{tabular}

Figure 2 Regression $(\beta)$ of alcohol use on demographic and lifestyle variables. Note. $\beta$ (beta): standardized regression coefficients, with standard errors in parentheses. Betas in bold font were significant at $\alpha=.01$ in the main sample and in the validation sample. Betas in regular black font were significant (at $\mathrm{a}=.01$ ) in the main sample but not in the validation sample. Betas in grey were not significant (at $\mathrm{a}=.01$ ). 
younger at alcohol initiation, onset of regular use, and first intoxication (betas between .12 and .22). This sex difference was significantly smaller in the young, than in the older participants (betas ranged between .12 and .20). Women mostly preferred wine $(\beta=.76)$, and among men, beer was the most popular beverage $(\beta=-.90)$.

High educational attainment, being a student, experiencing financial stress, and high degree of urbanization were associated with higher levels of alcohol use (betas between .06 and .36), while work-related stress, religiousness, and high BMI were related to lower levels of alcohol use (betas between -.03 and -.13). Initiation of smoking and cannabis were strongly, positively related to many aspects of alcohol use (betas between .07-.62), as well as to early age at alcohol initiation and age at first intoxication (betas between -.03 and -.17).

The proportion of variance explained by the demographic and lifestyle variables ranged between 5\% (urge to drink while relaxing) and $27 \%$ (age at onset regular drinking).

\section{Discussion}

A wealth of information on drinking patterns in the Dutch population was obtained from the analysis of multiple alcohol consumption variables in a large, population-based sample of adults, ranging in age between 18 and 97 years. Frequency and quantity of alcohol use, urges to drink, and indicators of more severe alcohol use were strongly associated with each other, but less strongly with age at initiation of alcohol use, onset of regular drinking, and first alcohol intoxication. With respect to effects of sex and age, we highlight the most important findings.

Alcohol consumption was high in men and women above age 55, suggesting that the trend of increased alcohol consumption in older Dutch adults is continuing [16]. Specifically, men and women (above age 55) drank more frequently than young adults. Excessive drinking ( $>14$ glasses of alcohol per week) was substantially more prevalent among women above age 55 than in the young adult age group. The prevalence of excessive drinking was lowest in women between ages 25-35 years, when they typically have children [59], and the high proportion of excessive drinking in elderly women may be explained by their having more opportunity to drink when their children enter adulthood. Increases in alcohol consumption in older adults may be due to the growing number of healthy life years, in combination with a higher average income, which has increased substantially over the past years in the elderly Dutch population [60,61]. Problematic alcohol use poses greater health risks for older adults than for younger individuals, since with increasing age, tolerance to the effects of alcohol declines. In addition, older adults may use medication that interacts negatively with alcohol, or suffer from chronic physical or psychological illness, such as diabetes or mood problems, that may be worsened by alcohol $[22,62]$.

Recent data suggest that the gap between male and female drinking is narrowing among young adults $[13,63,64]$. This trend is corroborated by the finding that among participants between 18-25 years old, women initiated alcohol use, started drinking regularly, and reported first alcohol intoxication at the same ages as men. Declining sex differences are mainly caused by increases in alcohol use in women, which may result from women having more freedom and financial independence. Drinking among women has also become more socially accepted than several decades ago, and young women nowadays may have fewer family responsibilities [65]. This trend has important consequences for public health, since women are more susceptible to the harmful effects of alcohol, such as liver and heart disease, than men [66].

Additionally, since the 1940s-50s, when the individuals in the highest age category were adolescents, alcohol initiation, onset of regular drinking, and first intoxication have been occurring at increasingly younger ages. This is in line with the increase in alcohol consumption in the Dutch population observed between 1950 and 1980, specifically of beer and imported wine $[67,68]$. The increase was likely due to strong economic growth and increases in international commerce during this period [69]. It should be noted, however, that the differences in age at alcohol initiation between young and old participants may be slightly overestimated due to retrospective reporting bias [70,71].

Besides age and sex, initiation of cigarette and cannabis use were the most striking risk factors for increased alcohol use, early alcohol initiation and age at first alcohol intoxication. These associations are likely explained by an underlying genetic vulnerability for substance use [72]. Individuals who use multiple substances often meet more criteria for abuse or dependence than those who only use a single substance. Moreover, using more than one substance is associated with increased health risks, due to the combined harmful effects of alcohol and cigarettes or cannabis $[73,74]$.

Some limitations of this study should be noted. Selfreport data may be imprecise due to memory errors or underreporting of substance use [75]. Moreover, 50\% of the sample were twins. Twins are born in all population groups, which makes having a twin in the family a suitable criterion for families to be included in a population-based sample [76]. Nevertheless, twin studies have been criticized since pregnancy and birth of twins carry increased risks and twins generally have a lower birth weight than singletons [77]. These are risk factors for behavioral problems, which are strongly related to alcohol use $[78,79]$. However, studies in several European countries 
have not observed differences in externalizing problems between twins and singletons during childhood and adolescence [80,81].

The participants in the $65+$ age group ranged in age between 65 and 97 years. As age increases, so does the risk of cognitive impairment, which may have lead to imprecise reports of alcohol use [22]. Moreover, although the cutoff for hazardous drinking based on the AUDIT questionnaire was lowered for the older participants, it may not be appropriate for this entire age range, since with increasing age, the ability to metabolize alcohol decreases $[22,62]$. Similarly, the CAGE questionnaire was developed in a sample of males under age 61 [48], and may therefore not be entirely appropriate for the oldest participants.

Excessive alcohol use was based on number of glasses consumed per week, and thus may have been somewhat inaccurate, since the criteria for excessive alcohol use are based on standard glasses of alcohol [45], which were not specifically reported in the survey.

Because of the large number of predictor variables, no other interaction effects were examined than between age and sex, while these may be relevant in the prediction of alcohol consumption. For example, those above age 55 years who seek treatment for alcohol use disorders are more often highly educated than treatment seekers below age 55 years [16]. Similarly, alcohol dependence is positively related to high education in women, but not in men [27].

To summarize, we observed that elderly Dutch men and women continued to drink alcohol more frequently than young adults, and excessive drinking was substantially more prevalent in elderly women than in young adult women. Until now, alcohol prevention campaigns have predominantly targeted adolescents and young adults, but the high levels of alcohol consumption among older adults warrant prevention and intervention campaigns aimed specifically at this age group [82]. Initiation of alcohol use, onset of regular drinking, and first alcohol intoxication occur at increasingly younger ages, and the gap that previously existed between men and women in age at alcohol initiation, age at onset of regular drinking, and age at first alcohol intoxication continues to close.

\section{Conclusions}

In the Netherlands, men and women above age 55 drink alcohol more frequently than young adults, and excessive drinking is substantially more prevalent in these women than in young adult women. Alcohol initiation, onset of regular drinking, and first alcohol intoxication occur at increasingly younger ages, and women continue to catch up to men with respect to when they initiate (regular) alcohol use and first get intoxicated. These trends have negative implications for public health, since women and older adults are particularly susceptible to the harmful effects of alcohol.

\section{Additional files}

Additional file 1: Table A1. Prevalence/distribution or mean and standard deviation of alcohol use indicators, by age and sex, in unweighted subjects.

Additional file 2: Table A2. Cross-validation of regression ( $\beta$ ) of alcohol use on demographic and lifestyle variables.

\section{Competing interests}

The authors declare that they have no competing interests.

\section{Authors' contributions}

LG and JvB collected the data, under supervision of JV, GW, MB, and DB. DB initiated the study. LG conducted the analyses and drafted the manuscript. JVB, JV, GW, MB, and DB commented on the manuscript. All authors read and approved the final manuscript.

\section{Acknowledgements}

This research was supported by the Addiction program of ZonMW (31160008): Genetic determinants of risk behavior in relation to alcohol use and alcohol use disorder; the European Research Council (ERC-230374): Genetics of Mental IIIness; the Netherlands Organization for Scientific Research (NWO 463-06-001): J.M. Vink is supported by ERC Starting Grant 284167

\section{Author details}

${ }^{1}$ Department of Biological Psychology, VU University Amsterdam, Amsterdam, The Netherlands. ${ }^{2}$ Neuroscience Campus Amsterdam, Amsterdam, The Netherlands. ${ }^{3}$ EMGO+ Institute for Health and Care Research, VU University Medical Center, Amsterdam, Amsterdam, The Netherlands.

Received: 17 July 2012 Accepted: 24 February 2013

Published: 8 March 2013

\section{References}

1. European Commission: EU citizen's attitudes towards alcohol. Brussels: European Commission; 2010.

2. Anderson P, Møller L, Galea G: Alcohol in the European Union: Consumption, harm and policy approaches. Copenhagen, WHO Regional Office for Europe; 2012:11

3. WHO-HFA: European health for all database. http://data.euro.who.int/hfadb/.

4. WHO: Global Status Report on Alcohol and Health 2011. Geneva, Switzerland: World Health Organization; 2011.

5. van Rooij AJ, Schoenmakers TM, van de Mheen D: Nationaal Prevalentie Onderzoek Middelengebruik 2009: De kerncijfers [National Prevalence Study on Substance Use 2009: Core Statistics]. Rotterdam, the Netherlands: IVO; 2011:13.

6. Substance Abuse and Mental Health Services Administration: State Estimates of Substance Use and Mental Disorders from the 2008-2009 National Surveys on Drug Use and Health. Rockville, MD: Substance Abuse and Mental Health Services Administration; 2011.

7. Nazareth I, Walker C, Ridolfi A, Aluoja A, Bellon J, Geerlings M, Svab I, Xavier M, King M: Heavy episodic drinking in Europe: a cross section study in primary care in six European countries. Alcohol Alcohol 2011, 46:600-606.

8. Ouwehand AW, Wisselink DJ, Kuijpers WGT, van Delden EB, Mol A: Kerncijfers Verslavingszorg 2010 [Figures Addiction health care 2010]. Houten, the Netherlands: LADIS / IVZ; 2011.

9. World Health Organization: Global Status Report on Alcohol and Health 2011. Geneva, Switzerland: World Health Organization (WHO); 2011.

10. Ooyen-Houben Van MMJ, Meijer RF, Brunt T, Laar Van M, Cruts AAN: National Drug Monitor - Year Report 2009. Utrecht, the Netherlands: Netherlands Institute of Mental Health and Addiction; 2010:149-178.

11. WHO: Global Health Risks: Mortality and Burden of Disease Attributable to Selected Major Risks. Geneva, Switzerland: WHO; 2009:21-22. 
12. Geels LM, Bartels M, van Beijsterveldt TCEM, Willemsen $G$, van der Aa N, Boomsma DI, Vink JM: Trends in adolescent alcohol use: effects of age, sex and cohort on prevalence and heritability. Addiction 2012, 107:518-527.

13. Van Laar M, Cruts AAN, Van Ooyen-Houben MMJ, Meijer RF, Croes EA, Brunt T, Ketelaars APM: National Drug Monitor - Year Report 2010. Utrecht, the Netherlands: Netherlands Institute of Mental Health and Addiction; 2011.

14. Netherlands Institute on Mental Health and Addiction: Studenten Alcoholgebruik [Students - Alcohol use]. http://www.alcoholinfo.nl/index.cfm? $a c t=e s i t e . t o n e n \& a=3 \& b=52 \& c=238$.

15. Zantinge EM, Van Laar MW: Hoeveel mensen gebruiken alcohol? [How many people use alcohol?]. Bilthoven: the Netherlands National Institute for Public Health and the Environment (RIVM); 2011.

16. Weingart S: Alcohol en ouderen in de verslavingszorg in Nederland (19992007) [Alcohol and the elderly in addiction health care in the Netherlands (1999-2007)]. Utrecht, the Netherlands: Netherlands Institute on Mental Health and Addiction; 2009.

17. Anthony JC, Echeagaray-Wagner F: Epidemiologic analysis of alcohol and tobacco use. Alcohol Res Health 2000, 24:201-208.

18. Falk D, Yi H, Hiller-Sturmhöfel S: An epidemiologic analysis of co-occurring alcohol and drug use and disorders. Alcohol Res Health 2008, 31:100-110.

19. Hingson RW, Zha W, Weitzman ER: Magnitude of and trends in alcoholrelated mortality and morbidity among U.S. college students ages 18-24, 1998-2005. J Stud Alcohol Drugs Suppl 2009, 16:12-20.

20. Carter AC, Brandon KO, Goldman MS: The college and noncollege experience: a review of the factors that influence drinking behavior in young adulthood. J Stud Alcohol Drugs 2010, 71:742-750.

21. Blazer DG, Wu LT: The epidemiology of at-risk and binge drinking among middle-aged and elderly community adults: National Survey on Drug Use and Health. Am J Psychiatry 2009, 166:1162-1169.

22. Blazer DG, Wu LT: The epidemiology of alcohol use disorders and subthreshold dependence in a middle-aged and elderly community sample. Am J Geriatr Psychiatry 2011, 19:685-694.

23. Savelkoul M, Verweij A, Zantinge EM: Alcoholgebruik: Zijn er verschillen naar sociaaleconomische status? [Alcohol use: Are there differences by socioeconomic status?]. Bilthoven, the Netherlands: National Institute for Public Health and the Environment (RIVM); 2011.

24. Schutten M, van den RJJM E, Knibbe RA: Onderzoeksrapportage Alcohol en Werk [Research report Alcohol and Profession]. The Hague, the Netherlands: Ministry of Social Affairs and Employment / Ministry of Health Welfare and Sport; 2003.

25. Maas Van Der E: Factsheet Alcohol en Werk [Factsheet Alcohol and Profession]. Woerden, the Netherlands: Netherlands Institute for Health Promotion (NIGZ); 2006

26. Moos RH, Brennan PL, Schutte KK, Moos BS: Older adults' coping with negative life events: common processes of managing health, interpersonal, and financial/work stressors. Int J Aging Hum Dev 2006, 62:39-59.

27. van MW L: Afhankelijkheid van alcohol: Zijn er verschillen naar sociaaleconomische status? [Alcohol dependency: Are there differences by socioeconomic status?]. Bilthoven, the Netherlands: National Institute for Public Health and the Environment (RIVM); 2010

28. Donath C, Grassel E, Baier D, Pfeiffer C, Karagulle D, Bleich S, Hillemacher T: Alcohol consumption and binge drinking in adolescents: comparison of different migration backgrounds and rural vs. urban residence-a representative study. BMC Publ Health 2011, 11:84.

29. Lawrence RJ, Fudge C: Healthy Cities in a global and regional context. Health Promot Int 2009, 24:i11-i18.

30. Statistics Netherlands: De geest uit de fles: Gaat kerkelijke betrokkenheid samen met drankgebruik? [The spirit out of the bottle: Does religious involvement co-occur with alcohol use?]. Voorburg/Heerlen, the Netherlands: Statistics Netherlands; 1999.

31. Aldridge-Gerry AA, Roesch SC, Villodas F, McCabe C, Leung QK, Da Costa M: Daily stress and alcohol consumption: modeling between-person and within-person ethnic variation in coping behavior. J Stud Alcohol Drugs 2011, 72:125-134.

32. Drerup ML, Johnson TJ, Bindl S: Mediators of the relationship between religiousness/spirituality and alcohol problems in an adult community sample. Addict Behav 2011, 36:1317-1320.

33. Willemsen MC, Feenstra D, van HJ K, Zantinge EM: Wat zijn de mogelijke oorzaken van roken? Which are the possible causes of smoking?]. Bilthoven, the Netherlands: National Institute for Public Health and the Environment (RIVM); 2011
34. Ministry of Security and Justice: Soft Drugs - Dutch Drugs Policy. http://www. government.nl/issues/alcohol-and-drugs/drugs.

35. Breslow RA, Smothers BA: Drinking Patterns and Body Mass Index in Never Smokers. Am J Epidemiol 2005, 161:368-376.

36. Sayon-Orea C, Martinez-Gonzalez MA, Bes-Rastrollo M: Alcohol consumption and body weight: a systematic review. Nutr Res 2011, 69:419-431.

37. Willemsen G, Vink JM, Abdellaoui A, Den Braber A, Van Beek JHDA, Draisma HHM, Van Dongen J, Geels LM, Van Lien R, EntD V't, et al: The Adult Netherlands Twin Register: 25 years of survey and biological data collection. Twin Res Hum Genet 2013, 16:271-81.

38. Koopmans JR, van LJP D, Boomsma DI, Goldbourt U, DeFaire U, Berg K: Smoking and Sports Participation. In Genetic Factors in Coronary Heart Disease. Dordrecht, The Netherlands: Kluwer; 1994:217-235.

39. Boomsma DI, Vink JM, van Beijsterveldt TC, de Geus EJ, Beem AL, Mulder EJ, Derks EM, Riese H, Willemsen GA, Bartels M, et al: Netherlands Twin Register: a focus on longitudinal research. Twin Res Hum Genet 2002, 5:401-406.

40. Boomsma DI, de Geus EJC, Vink JM, Stubbe JH, Distel MA, Hottenga JJ, Posthuma D, van Beijsterveldt TCEM, Hudziak JJ, Bartels M, Willemsen G: Netherlands Twin Register: From twins to twin families. Twin Res Hum Genet 2006, 9:849-857.

41. Statistics Netherlands: Beroepsbevolking; behaalde onderwijs naar herkomst geslacht en leeftijd [Working population; educational attainment by etnicity sex and age]. Voorburg/Heerlen the Netherlands: Statistics Netherlands; 2012.

42. Willemsen G, Posthuma D, Boomsma DI: Environmental factors determine where the Dutch live: Results from the Netherlands Twin Register. Twin Res Hum Genet 2005, 8:312-317.

43. Statistics Netherlands: Opleidingsniveau en stedelijkheid naar leeftijd 2008 en 1998 [Educational attainment and urbanization by age 2008 and 1998]. Heerlen/Noorburg, the Netherlands: Statistics Netherlands; 2008.

44. Statistics Netherlands: Gezondheid, leefstijl, zorggebruik; t/m 2009 [Health, lifestyle, health care use; until 2009]. Voorburg/Heerlen, the Netherlands: Statistics Netherlands; 2010

45. Institute of Alcohol Studies: IAS Fact Sheet - What is problem drinking? St Ives, UK: Institute of Alcohol Studies; 2002

46. Health Council of the Netherlands: Guidelines for a healthy diet 2006. The Hague, the Netherlands: Health Council of the Netherlands; 2006.

47. West RJ, Russell MAH: Pre-abstinence smoke intake and smoking motivation as predictors of severity of cigarette withdrawal symptoms. Psychopharmacology 1985, 87:334-336.

48. Ewing JA: Detecting alcoholism. JAMA: J Am Med Assoc 1984, 252:1905-1907.

49. van Beek JHDA, Kendler KS, de Moor MH, Geels LM, Bartels M, Vink JM, van den Berg SM, Willemsen G, Boomsma DI: Stable genetic effects on symptoms of alcohol abuse and dependence from adolescence into early adulthood. Behav Genet 2012, 42:40-56.

50. Babor TF, Higgins-Biddle JC, Saunders JB, Monteiro MG: AUDIT: The Alcohol Use Disorders Identification Test - Guidelines for Use in Primary Care. 2nd edition. Geneva: World Health Organization, Department of Mental Health and Substance Dependence; 2001.

51. SPSS Inc: PASW Statistics 18. Chicago, II: SPSS Inc; 2009.

52. Muthén LK, Muthén BO: Mplus User's Guide. Los Angeles, CA: Muthén \& Muthén; 2010

53. Rebollo I, de Moor MH, Dolan CV, Boomsma DI: Phenotypic factor analysis of family data: correction of the bias due to dependency. Twin Res Hum Genet 2006, 9:367-376.

54. Verweij A, Sanderse C, Lucht F: Scholing en opleiding: Wat is de huidige situatie? [Schooling and education: What is the current situation?]. Bilthoven: (RIVM) NIfPHatE; 2011.

55. Ministry of Education Culture and Science: Aantal studenten in hoger onderwijs als percentage van de bevolking van 18-25 jaar [Number of students in higher education as percentage of the 18-25 year old population]. http:// www.trendsinbeeld.minocw.nl/grafieken/3_1_1_3.php.

56. Arts K: Ontwikkelingen in kerkelijkheid en kerkbezoek (1999-2008) [Developments in religiousness and churchgoing (1999-2008)]. In Religie aan het begin van de 21ste eeuw [Religion at the beginning of the 21st century. Den Haag/Heerlen, the Netherlands: Statistics Netherlands; 2009.

57. Statistics Netherlands: Meeste rokers gestopt rond hun veertigste [Most smokers quit around age 40 years]. http://www.cbs.nl/nl-NL/menu/themas/ gezondheid-welzijn/publicaties/artikelen/archief/2007/2007-2204-wm.htm. 
58. Statistics Netherlands: Drugsgebruik; leeftijd en geslacht gebruiker [Drug use; age and sex user]. Voorburg/Heerlen, the Netherlands: Statistics Netherlands; 2010.

59. Statistics Netherlands: Leeftijd ouders bij geboorte kind, 1996 en 2000-2006 [Parental age at birth of child, 1996 and 2000-2006]. http://www.cbs.nl/nl-NL/ menu/themas/bevolking/cijfers/incidenteel/maatwerk/2388-wm.htm.

60. Van Den Berg Jeths A, Timmermans JM, Hoeymans N, Woittiez IB: Ouderen nu en in de toekomst: Gezondheid, verpleging en verzorging 2000-2020 [Elderly now and in the future: Health, nursing and care taking 2000-2020]. Bilthoven, the Netherlands: National Institute for Public Health and the Environment (RIVM); 2004.

61. Otten F, Bos W, Vrooman C, Hoff S: Armoedebericht 2006 [Poverty report 2006]. Voorburg, the Netherlands: Statistics Netherlands; 2006.

62. National Institute on Alcohol Abuse and Alcoholism: Older Adults. Bethesda, MD: Alcoholism NloAAa; 2013.

63. Statistics N: Focus on Health. London, UK: National Statistics; 2004.

64. Grucza RA, Norberg KE, Bierut LJ: Binge drinking among youths and young adults in the United States: 1979-2006. J Am Acad Child Adolesc Psychiatry 2009, 48:692-702.

65. Smith L, Foxcroft D: Drinking in the UK - An exploration of trends. UK: Joseph Rowntree Foundation; 2009.

66. National Institute on Alcohol Abuse and Alcoholism: Women and Alcohol. Bethesda, MD: National Institute on Alcohol Abuse and Alcoholism (NIAAA); 2011.

67. Dutch Institute for Alcohol Policy (STAP): Feiten en cijfers [Facts and figures]. http://www.stap.nl/nl/home/feiten-en-cijfers.html.

68. Karlsson T, Osterberg E: Alcohol Policies in EU Member States and Norway - A Collection of Country Reports. Helsinki, Finland: European Commission; 2006.

69. Smits JP: Economische ontwikkeling, 1800-1995 [Economical development, 1800-1995]. Voorburg/Heerlen, the Netherlands: Statistics Netherlands; 1999.

70. Sartor CE, Bucholz KK, Nelson EC, Madden PA, Lynskey MT, Heath AC: Reporting bias in the association between age at first alcohol use and heavy episodic drinking. Alcohol Clin Exp Res 2011, 35:1418-1425.

71. Johnson EO, Schultz L: Forward telescoping bias in reported age of onset: an example from cigarette smoking. Int J Methods Psychiatr Res 2005, 14:119-129.

72. Kendler KS, Schmitt E, Aggen SH, Prescott CA: Genetic and Environmental Influences on Alcohol, Caffeine, Cannabis, and Nicotine Use From Early Adolescence to Middle Adulthood. Arch Gen Psychiat 2008, 65:674-682.

73. National Institute on Alcohol Abuse and Alcoholism: Alcohol and Tobacco. Bethesda, MD: National Institute on Alcohol Abuse and Alcoholism; 2007.

74. National Institute on Alcohol Abuse and Alcoholism: Alcohol and Other Drugs. Bethesda, MD: National Institute on Alcohol Abuse and Alcoholism; 2008.

75. Blazer DG, Wu LT: Patterns of tobacco use and tobacco-related psychiatric morbidity and substance use among middle-aged and older adults in the United States. Aging Ment Health 2012, 16:296-304.

76. Martin N, Boomsma D, Machin G: A twin-pronged attack on complex traits. Nat Genet 1997, 17:387-392.

77. Levy F, Hay D, McLaughlin M, Wood C, Waldman I: Twin sibling differences in parental reports of $A D H D$, speech, reading and behaviour problems. J Child Psychol Psychiatry 1996, 37:569-578.

78. Ernst M, Moolchan ET, Robinson ML: Behavioral and neural consequences of prenatal exposure to nicotine. J Am Acad Child Adolesc Psychiatry 2001, 40:630-641.

79. Meyers JL, Dick DM: Genetic and Environmental Risk Factors for Adolescent-Onset Substance Use Disorders. Child Adolesc Psychiatr Clin N Am 2010, 19:465-477.

80. Pulkkinen L, Vaalamo I, Hietala R, Kaprio J, Rose RJ: Peer reports of adaptive behavior in twins and singletons: is twinship a risk or an advantage? Twin Res 2003, 6:106-118.

81. Robbers SC, Bartels M, van Oort FV, van Beijsterveldt CE, van der Ende J, Verhulst FC, Boomsma DI, Huizink AC: A twin-singleton comparison of developmental trajectories of externalizing and internalizing problems in 6- to 12-year-old children. Twin Res Hum Genet 2010, 13:79-87.

82. Netherlands Institute of Mental Health and Addiction: Alcohol preventie [Alcohol prevention]. http://www.trimbos.nl/onderwerpen/alcohol-en-drugs/ alcohol/preventie.

doi:10.1186/1471-2458-13-207

Cite this article as: Geels et al:: Increases in alcohol consumption in women and elderly groups: evidence from an epidemiological study. BMC Public Health 2013 13:207.

\section{Submit your next manuscript to BioMed Central and take full advantage of:}

- Convenient online submission

- Thorough peer review

- No space constraints or color figure charges

- Immediate publication on acceptance

- Inclusion in PubMed, CAS, Scopus and Google Scholar

- Research which is freely available for redistribution

Submit your manuscript at www.biomedcentral.com/submit 